\title{
ANÁLISIS DE ESTABILIDAD DE CULTIVARES DE MAÍZ (Zea mays L.) EN AMBIENTES DE COSTA RICA
}

\author{
Nevio A. Bonilla ${ }^{1}$
}

\section{RESUMEN}

Se establecieron 21 ensayos de adaptación y rendimiento de grano de cultivares de maíz de grano blanco y amarillo durante las dos épocas de siembra de 1997 y 1998 . Se realizó un análisis combinado y de estabilidad (AMMI) para los cultivares que estuvieron presentes en las localidades de Cañas, La Cruz, Santa Cruz, Upala, Guatuso, Los Chiles, Buenos Aires, Laurel, Pejibaye y Puriscal durante los períodos mencionados. Se analizaron los siguientes cultivares: Centella, Relámpago, 3031, A- 7573, DK-880 y Los Diamantes 8843 como testigo. El análisis de varianza mostró que las localidades La Cruz y Cañas presentaron el mayor rendimiento de grano (8,5 y $8,0 \mathrm{t} / \mathrm{ha}$, respectivamente) y las localidades de Santa Cruz, Pejibaye y Upala mostraron el menor rendimiento $(2,3,2,4$ y 2,4 t /ha respectivamente). El cultivar A-7573 mostró el mayor rendimiento promedio de grano (5,6 $t /$ ha) en todas las localidades evaluadas y el cultivar Los Diamantes 8843 presentó el menor rendimiento promedio (4,2 t/ha). El análisis AMMI de estabilidad para rendimiento de grano de los cultivares indicó que la interacción genotipo por ambiente obtenida con el análisis de varianza del combinado, obedece a que algunos de los genotipos estudiados presentan una interacción significativa $(p \leq 0,05)$ con los ambientes considerados. En este sentido, el análisis de componentes principales mostró que el cultivar Centella interaccionó de manera positiva con los diferentes ambientes $(2,06)$ y los cultivares A-7573 y DK-880 interactuaron de manera negativa $(-1,19$ y $-1,76)$. Por esta razón, se podría afirmar que los dos últimos requirieron de condiciones específicas para su buen desempeño, mientras que Centella se adaptó a un rango más amplio de ambientes. Así mismo, los cultivares Relámpago, 3031, y Los Diamantes 8843 interactuaron poco con el ambiente por lo que se consideran los más estables $(0,93,0,14$ y 0,59$)$.

Palabras clave: maíz, cultivares, estabilidad AMMI, ambientes.

\section{INTRODUCCIÓN}

La producción de maíz en la zona Centroamericana y del Caribe, se desarrolla en diversidad de ambientes que provocan en muchos casos bajos rendimientos. Los principales factores que afectan la producción del cultivo son la fertilidad y el manejo de los suelos, la deficiente distribución de las lluvias, el inadecuado manejo agronómico y la susceptibilidad o la resistencia de los diferentes genotipos a enfermedades e insectos.
Con el objeto de desarrollar cultivares de maíz estables en adaptación y con rendimiento adecuado a través de los ensayos regionales, se evalúa el comportamiento de variedades e híbridos provenientes de diferentes fuentes, los cuales son sembrados en un amplio rango de ambientes en todo el país. La información obtenida de estos ensayos permite la toma de decisión en la selección de cultivares adecuados para las diferentes regiones maiceras.

\footnotetext{
1 Instituto Nacional de Innovación y Transferencia en Tecnología Agropecuaria (INTA). Dirección de Investigación y Desarrollo Tecnológico, Departamento Investigación e Innovación. Apdo. 10094, San José, Costa Rica. Tel: (506) 231-2619; Fax: (506) 2960858. Email: nbonilla22 @yahoo.com
} 
Según Ordás (1995) la elección de unos objetivos apropiados es fundamental para desarrollar variedades e híbridos que sean superiores a los habituales en uso y que estén bien adaptados a la zona a la que se destinan. La correcta elección de objetivos debe basarse en un cuidadoso estudio de las características que se necesitan mejorar junto con una valoración precisa de los beneficios que el agricultor obtendrá al cultivar la nueva variedad.

De acuerdo con Córdoba et al. (1992) la evaluación experimental de maíz híbrido con relación a las variedades de polinización libre (V.P.L.) ha recibido mayor atención por parte de los fitomejoradores del área en la búsqueda de alternativas para aumentar la producción de maíz, particularmente en ambientes favorables. Estudios realizados por el CIMMYT (1989) en la región han determinado que los programas nacionales de maíz, han desarrollado en sus propios países proyectos de mejoramiento, con el fin de generar variedades e híbridos con mayor potencial de rendimiento y mejores características agronómicas.

Quemé y Fuentes (1992) consideran que el uso de germoplasma mejorado es una opción para evaluar la producción y productividad del cultivo; sin embargo, el éxito depende de la capacidad de adaptación de los genotipos a diferentes ambientes, por lo que se hace necesario hacer evaluaciones en diferentes localidades y años. Asimismo, Castañón et al. (1994) consideran que el manejo agronómico es el complemento indispensable para el buen desarrollo o comportamiento de un cultivo.

Shagún (1993) afirma que la evaluación de los genotipos en el tiempo y el espacio es una condición indispensable para estimar objetivamente su auténtico potencial agronómico y de rendimiento.

Pixley (1993) indica que un material nuevo además de adaptarse a una localidad determinada, debe superar a los ya existentes, para justificar la sustitución de éstos y la introducción de aquellos. De acuerdo con Ordás
(1995) el rendimiento (producción de grano seco por unidad de superficie) es la característica más importante en la mejora de maíz, así mismo es el objetivo más complejo con que tiene que trabajar el mejorador ya que está determinado por la expresión e interacción de numerosos genes que afectan todos los procesos vitales de la planta. Menciona además que la adaptación es una característica igualmente compleja dado que considera muchas y diferentes respuestas de la planta.

Córdoba (1990) sugiere que una evaluación del comportamiento de cultivares adaptados a los ambientes no favorables y favorables debe involucrar localidades cuya magnitud en la incidencia de factores adversos bióticos y abióticos contribuya a reducir la producción. Romagosa y Fox (1993) mencionan que la magnitud de las respuestas diferentes para rendimiento de genotipos evaluados en ambientes contrastantes puede ser fácilmente cambiada por efecto de la interacción genotipo-ambiente, máxime cuando la misma está influenciada por factores adversos de tipo biótico y abiótico.

Brizuela et al. (1992) indican que al utilizar modelos de estabilidad, se confirma el comportamiento de la interacción genotipo por ambiente y llevan a conclusiones muy positivas para poder seleccionar materiales de mejor rendimiento. Romagosa y Fox (1993) afirman que la interacción Genotipo x Ambiente es la expresión diferencial a lo largo de los ambientes, así mismo ésta reduce la asociación entre los valores fenotípicos y genotípicos y pueden causar que las selecciones provenientes de un ambiente se comporten pobremente en otro, forzando a los fitomejoradores a examinar la adaptación genotípica. Destacan además que es importante medir la interacción genotipo $x$ ambiente para determinar una estrategia de mejoramiento adecuada para liberar genotipos con la adaptación acorde a los ambientes de interés.

Allard (1960) según Romagosa y Fox (1993) describió la complejidad biológica señalando que la interacción genotipo $x$ ambiente se da cuando virtualmente todos 
los efectos fenotípicos no están relacionados con el gen de una manera simple. Por el contrario resultan de una cadena de reacciones fisicoquímicas y las interacciones iniciadas por los genes pero conduciendo a través de complejas cadenas de eventos, controladas o modificadas por otros genes y el ambiente externo, hacia el fenotipo final. Romagosa y Fox (1993) indican que cuando en un análisis de variancia la interacción genotipo $x$ ambiente es significativa, los efectos principales deben ser interpretados con cuidado y la naturaleza de la interacción examinada, ya que las medias a menudo enmascaran casos donde los genotipos se comportan bien o pobremente en subconjuntos de sitios.

El objetivo de este trabajo fue determinar el comportamiento agronómico y adaptabilidad de los diferentes híbridos y variedades comerciales y experimentales de maíz bajo las diferentes condiciones edáficas y climáticas de las regiones maiceras del país.

\section{MATERIALES Y MÉTODOS}

Para este estudio se consideraron los datos de los ensayos regionales de maíz llevados a cabo durante las dos épocas de siembra de los años 1997 y 1998, de manera que mediante la metodología de análisis combinado y de estabilidad de genotipos (AMMI) (Crossa et al. 1990); Crossa et al. (1991) trataron de determinar los genotipos de maíz más estables durante dicho período. Las localidades consideradas, fueron: Cañas, La Cruz, Santa Cruz, Upala, Guatuso, Los Chiles, Buenos Aires, Potrero Grande, Laurel, Pejibaye y Puriscal. En el Cuadro 1 se presentan los datos de clima promedio de las localidades mencionadas anteriormente.

El trabajo correspondiente a 1997 se inició en junio de ese año y finalizó en febrero de 1998 y el correspondiente a 1998 se inició en mayo de ese año y finalizó en febrero de
1999. El diseño experimental utilizado en ambos períodos fue bloques completos al azar con cuatro repeticiones y la unidad experimental consistió de parcelas con cuatro surcos de $5 \mathrm{~m}$ de largo distanciados $0,75 \mathrm{~m}$ entre sí, donde se sembraron tres semillas por cada sitio de siembra distanciados $0,5 \mathrm{~m}$ y se raleó a dos plantas a los 15 días después de la siembra. La parcela útil estuvo constituida por los dos surcos centrales para un área de 7,5 m cuadrados. Dicho trabajo se llevó a cabo en dos ciclos de siembra durante los años 1997 y 1998, de tal manera que se consideraron tanto la primera como la segunda época de siembra de cada año.

Las variables y métodos de evaluación utilizados para cada uno de los ensayos de campo fueron:

\section{Comportamiento agronómico}

1. Número de plantas emergidas (dato de observación, cuando amerite).

2. Días a floración masculina (número de días desde la siembra, 50 \% anteras con polen).

3. Días a floración femenina (número de días desde la siembra , 50 \% anteras con polen).

4. Altura de planta (en $\mathrm{cm})$.

5. Altura de mazorca (en $\mathrm{cm}$ ).

6. Cobertura de mazorca (escala uno a cinco, donde uno es mayor cobertura).

7. Aspecto de mazorca (uno a cinco, donde uno es óptimo y cinco muy deficiente).

8. Acame de raíz (número de plantas).

9. Acame de tallo (número de plantas).

\section{Problemas de plagas y enfermedades}

1. Número de mazorcas podridas.

2. Enfermedades de follaje (escala uno a cinco, donde uno es $100 \%$ sanidad y cinco es $100 \%$ enfermo). 


\section{Rendimiento}

1. Número total de mazorcas.

2. Número total de plantas.

3. Peso de campo.

4. Relación grano /mazorca.

5. Rendimiento de grano al $14 \%$ humedad.

Los cultivares analizados en este estudio fueron:

Primera época de siembra 1997: HS-6, G.Morales, Trueno, Centella, Relámpago, Huracán, X 1404G, A-7573, Tornado, Los Diamantes 8843, Deifín, A-7597, DK 880, J.Saénz.

Segunda época de siembra 1997: HS-6, DK-880, Huracán, DK-888, 30M48, A-7573, Relámpago, Centella, Trueno, Tornado, X1404 G, Deifín, Los Diamantes 8843, A-7597.

Primera época de siembra 1998: $\mathrm{H}-94$, H-92, H-203, Centella, Relámpago, 3031, 30M48, A-7573, 7VM 1012, Los Diamantes 8843, DK-880, DK-888 A.

Segunda época de siembra 1998: Relámpago, 3031, 30M48, A-7573, 7VM 1012, Los Diamantes 8843, DK-880, DK-888 A, Local, Ciclón, Sintético, CB-HS9, CB-
HS9GM14, CB-HS9GM7, HS-5G, HS-8, C220, C-385.

Para el análisis de los datos se utilizó el análisis de varianza por localidad, empleando la prueba de rango múltiple de Duncan al $p=$ 0,05 , y se efectuó un análisis combinado de todos los ensayos. Además se realizó un análisis mediante el índice de selección para determinar los mejores genotipos en cada experimento. Este índice se basa en la intensidad y en la meta de selección para las variables evaluadas en cada ensayo, de manera que se integren los criterios técnicos del mejorador en un solo valor numérico (Barreto et al. 1991). De acuerdo con esta metodología mientras más pequeño es el valor del índice más cerca se encuentra el genotipo de los criterios deseados por el usuario, y es por tanto superior. Además se realizó un análisis de estabilidad AMMI (Crossa et al. 1990; Crossa et al. 1991) que determina la interacción genotipo $x$ ambiente significativa obtenida en el análisis de variancia del combinado para $21 \mathrm{am}$ bientes correspondientes al período 1997-98 y para seis genotipos del total que estuvieron en dichos ambientes, esto debido a que los mismos son los únicos presentes en todos los ambientes considerados. Dichos genotipos fueron Centella, Relámpago, DK-880, A-7573, Los Diamantes 8843 y 3031.

Cuadro 1. Características climáticas de los sitios estudiados durante el año 1997 y 1998 . Valores promedio anual.

\begin{tabular}{lrlccc}
\hline Sitio & $\begin{array}{c}\text { Altitud } \\
(\mathbf{m s n m})\end{array}$ & $\begin{array}{c}\text { Zona de vida } \\
\text { (Holdrige) }\end{array}$ & $\begin{array}{c}\text { Temperatura } \\
\left.\mathbf{(}{ }^{\circ} \mathbf{C}\right)\end{array}$ & $\begin{array}{c}\text { Precipitación } \\
(\mathbf{m m})\end{array}$ & $\begin{array}{c}\text { Humedad } \\
\text { relativa (\%) }\end{array}$ \\
\hline Cañas & 255 & Bos. Trop.Seco & 28,9 & 1.630 & 80 \\
La Cruz & 337 & Bos.Hum. Trop. & 30,0 & 2.573 & 85 \\
Santa Cruz & 49 & Bos. Trop.Seco & 28,5 & 1.640 & 80 \\
Upala & 46 & Bos. Hum. Trop & 24,6 & 2.295 & 90 \\
Guatuso & 50 & Bos.Hum. Trop. & 26,0 & 2.780 & 90 \\
Los Chiles & 43 & Bos. Hum. Trop & 25,7 & 2.391 & 90 \\
Buenos Aires & 418 & Transición & 24,9 & 3.580 & 85 \\
Potrero Grande & 183 & Transición & 26,5 & 2.370 & 85 \\
Pejibaye & 38 & Transición & 27,2 & 3.150 & 90 \\
Laurel & 43 & Bos. Hum. Trop. & 24,9 & 2.620 & 85 \\
Puriscal & 1.105 & Bos. Hum. Prem. & 20,3 & 2.564 & 80 \\
& & Bajo & & \\
\hline
\end{tabular}




\section{RESULTADOS Y DISCUSIÓN}

\section{Análisis combinado de localidades}

\section{Ensayos 1997}

De acuerdo a los resultados obtenidos del análisis combinado de los ensayos establecidos en la primera época de 1997 los cultivares HS-6, A-7573, A-7597, Tornado, Trueno, Huracán y Relámpago presentaron los mayores rendimientos por área, a saber 8,3, 7,9, $7,4,8,5,7,7,7,7$ y 7,0 t/ha respectivamente. Las variedades criollas J. Saénz y G. Morales, así como el testigo local presentaron los menores rendimientos $(5,9,5,5$ y 5,6 t/ha respectivamente).Los porcentajes de pudrición de mazorca oscilaron entre $2,5 \%$ y $47,6 \%$ dependiendo de la localidad y de las condiciones en que se desarrolló el ensayo. Los cultivares A-7597, Centella, G. Morales, J.Saénz, Local, Relámpago mostraron los valores más altos de pudrición de mazorca. Las localidades en las que se dieron los porcentajes más altos de pudrición fueron: Los Chiles, Santa Cruz, Cañas (Estación Experimental Enrique Jiménez Núñez) y Potrero Grande, siendo estos $15,2,28,15,5$ y $8,5 \%$ respectivamente.

En el análisis combinado para la segunda época, los rendimientos más altos correspondieron a los cutivares 30M48, Tornado, Huracán, A-7597 y HS-6, a saber 4,0, 3,8, 3,8, 3,7 y 3,6 t/ha respectivamente. No hubo diferencias significativas para las variables acame de tallo y cobertura de mazorca. El cultivar DK-880 tuvo la menor altura de planta y mazorca; mientras que el híbrido 30M48 presentó la mayor altura de mazorca y los híbridos Tornado, Deifin, A-7597, Relámpago, Huracán, HS6 y 30M48 mostraron la mayor altura de planta. El aspecto de mazorca para los híbridos X-1404G, HS-6, DK-880 y Local fue 2,5 , y para los híbridos Relámpago y Deifín el aspecto fue de 3,5 .

Los cultivares Los Diamantes 8843, A7573 y 3031 tuvieron los índices más bajos con respecto a los demás cultivares. Esto in- dica que dichos cultivares presentaron los valores más adecuados para las variables consideradas en el análisis de selección. (Los Diamantes 8843 0,1 y 12,00; A-7573 1,4 y $11,00 ; 30311,9$ y 10,60$)$.

No se presentaron diferencias significativas para las variables acame de tallo y cobertura de mazorca. La menor altura de planta y mazorca fue para el cultivar D-880; mientras que la mayor altura de mazorca fue para el híbrido 30M48 y los híbridos Tornado, Deifín, A-7597, Relámpago, Huracán, HS6 y 30M48 mostraron la mayor altura de planta. Los híbridos X-1404G, HS-6, D-880 y Local tuvieron un aspecto de mazorca de 2,5 y los híbridos Relámpago y Deifín presentaron un aspecto de 3,5 .

\section{Ensayos 1998}

Los híbridos 7VM-1012, 30M48, 3031 y A-7573 mostraron los mayores rendimientos en las localidades establecidas durante la primera época de siembra, la cual se ubica en el período comprendido entre mayo y setiembre. También es importante destacar que los híbridos Centella, H-94, H-92 y H-203 dieron los rendimientos más bajos para las ocho localidades combinadas. Con respecto al rendimiento por localidad, al analizar el desempeño de las localidades se puede señalar que en La Cruz, Cañas, Los Chiles y Guatuso ocurrieron las condiciones más favorables para el desarrollo del cultivo y la localidad Potrero Grande presentó las condiciones más desfavorables.

Los cultivares 30M48, 7VM-1012 y Diamantes 8843 tuvieron las mayores alturas de planta $(238,8,226,0$ y $221,8 \mathrm{~cm}$ respectivamente. Así mismo los híbridos H-94, H92, H203 y Centella mostraron las menores alturas de planta $(187,6,184,3,186,2$ y $182,6 \mathrm{~cm}$ respectivamente). En cuanto a la altura de mazorca los cultivares 30M48, 7VM-1012 y Diamantes 8843 dieron los menores valores con relación a esta variable, además los híbridos $\mathrm{H}-94, \mathrm{H} 92, \mathrm{H}-203$ y Centella presentaron 
las menores alturas de mazorca $(88,7,82,7$, 85,3 y $80,6 \mathrm{~cm}$ respectivamente).

Los porcentajes de pudrición de mazorca ocurrieron en los híbridos H-203, Centella, A7573, Relámpago, Diamantes 8843, H-92 con valores de $18,8,17,3,16,9,15,6,14,9$ y 14,0 $\%$ respectivamente.

Los otros cultivares estuvieron por debajo del parámetro de $14 \%$ de humedad de grano. El híbrido $30 \mathrm{M} 48$ mostró el porcentaje más bajo de pudrición de mazorca (8,4\%). Es importante señalar que en las localidades Upala, Potrero Grande y Guatuso se presentaron los mayores porcentajes de pudrición $(20,4$, 19,6 y $18,4 \%$ respectivamente) y en las localidades Cañas y Los Chiles los porcentajes más bajos (4,9 y 7,7\% respectivamente).

Los híbridos 7VM-1012 y DK-888 A mostraron los mayores porcentajes de mala cobertura de mazorca, a saber 39,1 y $23,6 \%$ respectivamente. Los cultivares DK-880 y Diamantes 8843 mostraron los porcentajes de mala cobertura más bajos $(7,1$ y $9,0 \%$ respectivamente). El mayor porcentaje de mala cobertura fue para la localidad de Los Chiles $(26,5 \%)$ y los menores porcentajes para las localidades de Laurel, Cañas y Puriscal $(7,4$, 10,6 y $13,9 \%$ respectivamente).

El índice de selección indica que los menores valores de índice calculado fueron para los cultivares 7VM1012, DK-888 y Relámpago . Las variables que se tomaron en cuenta fueron: rendimiento de grano y porcentaje de pudrición de mazorca. (7VM1012 2,7, 7,4, 11,5; DK-888 A, 4,1, 6,1, 9,8; Relámpago 4,6, 5,5, 15,6).

En las localidades evaluadas en la segunda época los cultivares CB HS9G, CB HS9GM-7, CB HS9GM-14, CB HS8G, CB HS5G, 30M48, C-385, Diamantes 8843, 7VM-1012 y C-220 presentaron los mayores rendimientos. Los cultivares Local, Relámpago, Sintético y 3031 tuvieron los rendimientos más bajos. Las condiciones más adecuadas para el desarrollo de los cultivares en estudio se dan en las localidades La Cruz y Cañas , no así Buenos Aires (5,3, 4,8 y 2,2 t/ha respectivamente). Los cultivares CB HS9GM14, CB HS9GM7, Ciclón, CB HS9G, 3031 y 7VM1012 mostraron las mayores alturas de planta y mazorca. Así mismo los híbridos A7573, DK-880, C-220, C-385, Relámpago y 3031 mostraron las menores alturas de mazorca y planta.

En cuanto a la pudrición de mazorca los cultivares 3031, Sintético, CB HS9GM7, CB HS5G, CB HS8G, C-220 y C-385 presentaron los valores más bajos de porcentaje de pudrición. Así mismo, los cultivares CB HS9GM14, DK-880, Diamantes 8843, DK-888 A, Local y A-7573 mostraron los valores más altos de pudrición de mazorca. Es importante indicar que de los 18 cultivares evaluados solamente seis genotipos tuvieron valores superiores al $14 \%$. De los restantes 12 cultivares se determinó que en siete de ellos los valores de pudrición fueron inferiores al $10 \%$.

\section{Análisis de estabilidad 1997-1999}

Los Cuadros 2, 3, 4 y 5 corresponden al análisis de estabilidad de los genotipos seleccionados para este estudio en las localidades consideradas para las épocas de siembra de 1997 y 1998. En el Cuadro 2 del análisis de varianza de los ambientes y genotipos se evidencia una diferencia significativa para la interacción genotipo $x$ ambiente. En el Cuadro 3 se presenta el análisis de componentes principales, donde se muestran los puntajes asignados por el análisis AMMI a los seis genotipos seleccionados para los 21 ambientes que se tomaron en cuenta en este estudio.

Los valores positivos de puntaje fueron para los genotipos Centella, Relámpago y 3031 y los negativos para los genotipos A7573, DK-880 y Los Diamantes 8843, pero muy cercanos a cero. Esto indica que los primeros tuvieron una alta interacción con los ambientes considerados y que los últimos interactúan muy poco. 
Cuadro 2. Análisis de varianza incluyendo regresiones FINLAYWILKINSON. Análisis estabilidad 97-99 para seis genotipos y 21 ambientes con tres repeticiones.

\begin{tabular}{lrrrl}
\hline \multicolumn{1}{c}{ Fuente } & G. L. & $\begin{array}{c}\text { Suma } \\
\text { cuadrados }\end{array}$ & $\begin{array}{c}\text { Cuadrados } \\
\text { medios }\end{array}$ \\
\hline AMBIENTE & 20 & 169,23402 & 8,46170 & 0,2240276 \\
BLOQUE & 42 & 270,69083 & 6,44502 & 0,1038586 \\
GENOTIPO & 5 & 8,09178 & 5,61836 & 0,3331183 \\
G X A & 100 & 812,63947 & 8,12639 & $0,0010562^{* *}$ \\
REGRCONJ & 1 & 35,91232 & 35,91232 & $0,0071596^{* *}$ \\
REGRGEN & 4 & 87,19100 & 21,79775 & $0,0017152^{\text {** }}$ \\
REGRAMB & 19 & 128,44729 & 6,76038 & 0,1347035 \\
RESIDUAL & 76 & 561,08886 & 7,38275 & $0,0109760^{*}$ \\
ERROR & 210 & $1.022,41417$ & 4,86864 & \\
TOTAL & 377 & $2.303,07027$ & 6,10894 & \\
\hline
\end{tabular}

Regresión conjunta K = -0,1689796E+01

Cuadro 3. Análisis de componentes principales de genotipos para desviaciones de eje uno y análisis de estabilidad 97-99 para seis genotipos y 21 ambientes con tres repeticiones.

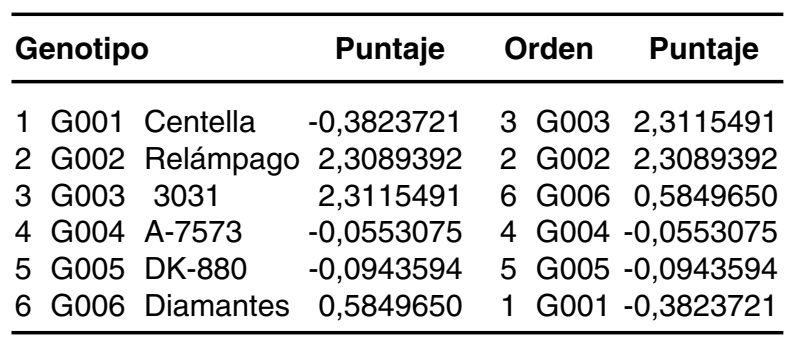

En el Cuadro 4 se muestra el análisis de componentes principales para los 21 ambientes considerados, donde se presentan los puntajes asignados por el análisis AMMI para cada ambiente. Es importante señalar que los ambientes interactuaron con los genotipos de manera positiva y negativa, destacándose el ambiente 11 (Upala) con el valor positivo más alto y los ambientes 13 (Cañas B) y 14 (Puriscal 98) con los puntajes negativos mayores. En el Cuadro 5 se muestra el análisis de varianza para el análisis de componentes principales donde se indica que exista alta significancia para los componentes PCA1 (Análisis de Componentes Principales 1) y PCA2 (Análisis de Componentes Principales 2).
Este resultado fundamenta el considerar ambos componentes para la elaboración del gráfico biplot (Figura 1) que explique la interacción genotipo $\mathrm{x}$ ambiente y la estabilidad de los genotipos seleccionados.

Cuadro 4. Análisis de componentes principales de ambientes para desviaciones del eje uno y análisis estabilidad 97-99 para seis genotipos y 21 ambientes con tres repeticiones.

\begin{tabular}{llrrrr}
\hline & Ambientes & Puntaje & Orden & Puntaje \\
\hline 1 & La Cruz & $-0,1599025$ & 11 & 0011 & 1,4299837 \\
2 & Los Chiles & 0,0389437 & 19 & 0019 & 0,9794404 \\
3 & Guatuso & $-0,3287747$ & 18 & 0018 & 0,9342870 \\
4 & Santa Cruz & 0,6367412 & 4 & 0004 & 0,6367412 \\
5 & Cañas & 0,5907524 & 5 & 0005 & 0,5907524 \\
6 & P. Grande & $-0,9055527$ & 12 & 0012 & 0,2899712 \\
7 & Laurel & $-0,8016404$ & 2 & 0002 & 0,0389437 \\
8 & Sabana & $-0,5237728$ & 15 & 0015 & 0,0199429 \\
9 & Pejibaye & $-0,2185387$ & 16 & 0016 & 0,0198643 \\
10 & La Cruz B & $-0,0145219$ & 10 & 0010 & $-0,0145219$ \\
11 & Upala & 1,4299837 & 21 & 0021 & $-0,0701357$ \\
12 & Laurel B & 0,2899712 & 1 & 0001 & $-0,1599025$ \\
13 Cañas B & $-1,2781258$ & 9 & 0009 & $-0,2185387$ \\
14 Puriscal 98 & $-1,3109956$ & 3 & 0003 & $-0,3287747$ \\
15 Guatuso 98 & 0,0199429 & 8 & 0008 & $-0,5237728$ \\
16 Laurel 98 & 0,0198643 & 17 & 0017 & $-0,5307173$ \\
17 & Cañas 98 & $-0,5307173$ & 7 & 0007 & $-0,8016404$ \\
18 La Cruz 98 & 0,9342870 & 6 & 0006 & $-0,9055527$ \\
19 Upala 98 & 0,9794404 & 20 & 0020 & $-0,9483817$ \\
20 & P.Grande 98 & $-0,9483817$ & 13 & 0013 & $-1,2781258$ \\
21 Buenos Aires 98 & $-0,0701357$ & 14 & 0014 & $-1,3109956$ \\
\hline
\end{tabular}


Cuadro 5. Análisis de varianza para análisis de componentes principales de desviaciones a partir de la media general. Análisis de estabilidad 97-98 para seis genotipos y 21 ambientes con tres repeticiones.

\begin{tabular}{|c|c|c|c|c|}
\hline Fuente & GL & $\begin{array}{c}\text { Suma } \\
\text { Cuadrados }\end{array}$ & $\begin{array}{l}\text { Cuadrados } \\
\text { Medios }\end{array}$ & Probabilidad \\
\hline PRUEBA & 125 & 1009,96527 & 8,07972 & $0,0006155^{\star \star \star}$ \\
\hline PCA 1 & 26 & 374,62945 & 14,40882 & $0,0000088^{\star * \star}$ \\
\hline PCA 2 & 24 & 260,35744 & 10,84823 & $0,0013898^{* *}$ \\
\hline RESIDUAL & 75 & 374,97838 & $3 \quad 4,99971$ & 0,4326093 \\
\hline BLOQUE & 42 & 270,69083 & 6,44502 & 0,1038586 \\
\hline ERROR & 210 & 1022,41417 & 4,86864 & \\
\hline TOTAL & 377 & 2303,07027 & 6,10894 & \\
\hline
\end{tabular}

En la Figura 1 se presentan los resultados obtenidos utilizando los datos del análisis de componentes principales, particularmente del PCA1 (Análisis de Componentes Principales 1) que explica un alto porcentaje de la variación debida a la interacción genotipo $\mathrm{x}$ am-
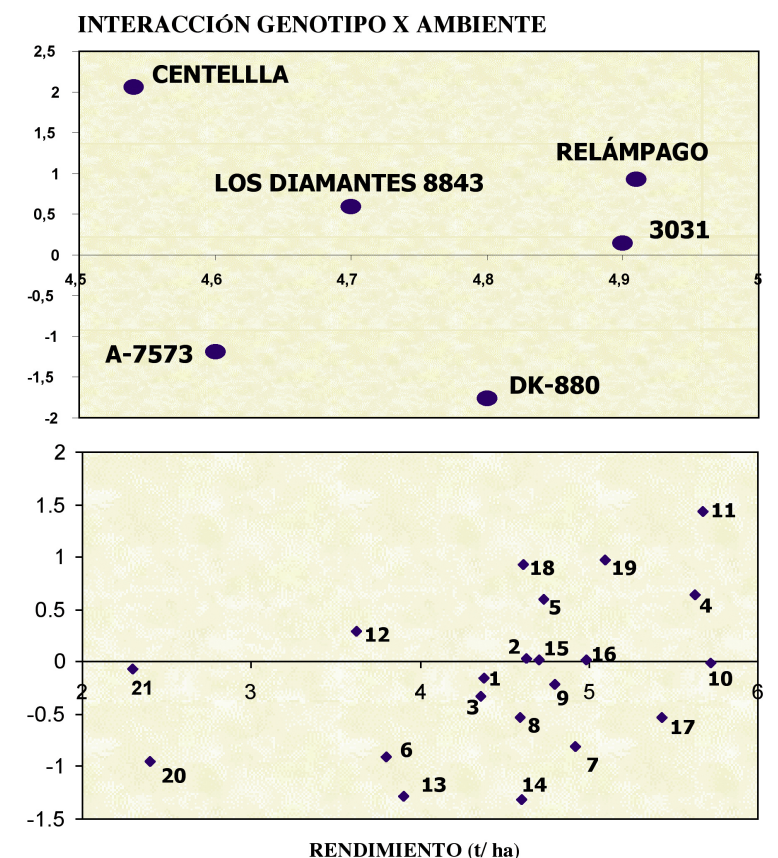

Figura 1. Medias de rendimiento puntuaciones del primer eje del componente principal de seis cultivares de maíz de grano blanco y 21 ambientes. 1997-1999.

1: La Cruz, 2: Los Chiles, 3: Guatuso, 4: Santa Cruz, 5: Cañas, 6: Potrero Grande, 7: Laurel, 8: Sabana, 9: Pejibaye, 10: La Cruz b, 11: Upala, 12: Laurel b, 13: Cañas b, 14: Puriscal 98, 15: Guatuso 98, 16: Laurel 98, 17: Cañas 98, 18: La Cruz 98, 19: Upala 98; 20: Potrero Grande 98 ; 21: Buenos Aires 98 biente. Se incluyen en la Figura 1 datos para 21 ambientes, así como los genotipos. Los genotipos más estables fueron Los Diamantes 8843 y 3031 . También los cultivares Centella y Relámpago presentaron una interacción positiva con los ambientes y los genotipos A-7573 y DK-880 interactuaron negativamente con la mayoría de los ambientes. Los ambientes que interactuaron poco con los genotipos fueron: 18 ( La Cruz 98), 15 (Guatuso 98),11 (Upala), 9 (Pejibaye) y 14 (Puriscal 98).

Estos resultados dejan ver que los genotipos más estables son Los Diamantes 8843 y 3031 y poseen una gran capacidad de adaptación a los diferentes ambientes en que estuvieron. Por el contrario los cultivares Centella y Relámpago parecieran más adaptados a condiciones de buenos ambientes y los genotipos A-7573 y DK-880 no se adaptan bien en condiciones de ambientes adversos o menos favorables.

Las localidades mencionadas como de poca interacción con los genotipos poseen ambientes que aportan poco a la distinción entre genotipos, no así los ambientes con interacciones positivas y negativas, ya que de una $u$ otra forma permiten diferenciar entre genotipos de amplio y reducido espectro de adaptación.

\section{CONCLUSIONES}

El análisis de componentes principales mostró que el cultivar Centella interaccionó de manera positiva con los diferentes ambientes $(2,06)$ y los cultivares A-7573 y DK-880 interaccionaron de manera negativa $(-1,19$ y $1,76)$. Por esta razón, se podría afirmar que los dos últimos requieren de condiciones específicas para su buen desempeño, mientras que Centella se adapta a un rango más amplio de ambientes. Así mismo, los cultivares Relámpago, 3031, y Los Diamantes 8843 interactúan poco con el ambiente por lo que se consideran los más estables $(0,93,0,14 \mathrm{y}$ $0,59)$. El análisis de los ambientes indica que 
localidades como Los Chiles, Buenos Aires y Laurel no aportan mucha interacción con los genotipos considerados, por lo que su validez como sitios para establecer diferencias entre ellos sería difícil de justificar. Sin embargo otros sitios tienen un comportamiento variable en los dos años considerados, por lo que sería importante correlacionar los datos con las condiciones climáticas específicas de cada ambiente para explicar más adecuadamente las tendencias de estos sitios de evaluación.

\section{AGRADECIMIENTO}

Al Técnico Dagoberto Espinoza Sánchez por su colaboración en el trabajo de montaje y evalución de los ensayos.

\section{LITERATURA CITADA}

Barreto, H.J.; Bolaños, J.A.; Córdova, H.S. G. Programa de índice de selección. Guía para la operación del software. Manual de Capacitación Regional. CIMMYT. 27 p.

Brizuela, L.; Dubón, T.; Campos, P.; Reyes, R. 1992. Evaluación de variedades sintéticas de maíz (Zea mays L.) en once ambientes de Centro América. In: J. Bolaños; G. Saín; R. Urbina; H. Barreto. eds. Síntesis de Resultados Experimentales de 1991 CIMMYT-PRM. p: 43-58.

Castañón, G.; Rodríguez, F. ; Sierra, M.; Castillo, R; Tosquy, O.; Barrón, S. 1994. Respuesta del maíz (Zea mays L.) a cinco factores de estudios (Híbrido, densidad de población, Nitrógeno, Fósforo y Potasio). In: Memoria XL Reunión Anual del PCCMCA. Costa Rica.

Centro Internacional para el Mejoramiento de Maíz y Trigo (CIMMYT). 1989. Programa Regional de Maíz de CIMMYT para Centro América, Panamá y el Caribe.

Córdoba, H.S.; Barreto, H.S.; Crossa, J. 1992. Impacto del Desarrollo de Híbridos de Maíz en Centro América: Confiabilidad de las Ganan- cias en Rendimiento sobre el Genotipo H5 y consideraciones para la Selección de Testigos Regionales. In: Síntesis de Resultados Experimentales 1992. Programa Regional de Maíz (PRM). p: 3-10 .

Córdoba, H.S. 1990. Estimación de parámetros de estabilidad para determinar la respuesta de híbridos de maíz (Zea mays L.) a ambientes contrastantes de Centroamérica, Panamá y México. In: Memoria XXXVL Reunión PCCMCA. San Salvador.

Crossa, J; Fox, P.N.; Pfeiffer, W.H.; Rajaram, S.; Gauch, Jr. H.G. 1991. AMMI adjustment for statistical analysis of an international wheat yield trial. Theoretical and Applied Genetics 81: 27-37.

; Gauch, Jr.; H.G.; Zobel, R.W. 1990. Additive Main Effects and Multiplicative Interaction Analysis of two international maize cultivar trials. Crop Science 30 (3):493-500.

Ordas, A. 1995. Mejora genética del maíz. Curso Superior de Mejora Genética Vegetal. Instituto Agronómico Mediterráneo de Zaragoza. Centro Internacional de Altos Estudios Agronómicos. 25 p.

Pixley, A.L. 1993. Informe final de ensayos presentado a la Unidad de Planificación de Investigaciones Agrícolas. $27 \mathrm{p}$.

Queme, J.L.; Fuentes, R.M. 1992. Evaluación de híbridos de maíz (Zea mays L.) de grano blanco y amarillo en diferentes ambientes de México, Centro América y El Caribe y Venezuela. In: Síntesis de Resultados Experimentales 1991. Programa Regional de Maíz (PRM). 283 p.

Romagosa, I.; Fox, P.N. 1993. Genotype-Environment Interaction and adaptation. In: M.D. Hayward; N.O. Bosemark; I. Romagosa. eds. Plant Breeding. Principles and Prospects. C.I.H.E.A.M. Zaragoza, Spain. Chapman \& Hall. London. p. 373-390.

Shagun, J. 1993. Evaluación de genotipos en serie de experimentos: diferencias en parámetros genéticos generados en dos modelos. In: Memoria XXXIX Reunión Anual PCCMCA. Guatemala. 